\title{
Simulations of some Doubly Stochastic Poisson Point Processes
}

\author{
B. Picinbono \\ Laboratoire des Signaux et Systèmes ${ }^{1}$, \\ Supélec, Plateau de Moulon, 91190 Gif-sur-Yvette, France
}

Tel: (33)-(1)-69.85.17.40, Email : bernard.picinbono@lss.supelec.fr July 2011

\begin{abstract}
Computer simulations of point processes are important either to verify the results of certain theoretical calculations that can be very awkward at times, or to obtain practical results when these calculations become almost impossible. One of the most common methods for the simulation of nonstationary Poisson processes is random thinning. Its extension when the intensity becomes random (doubly stochastic Poisson processes) depends on the structure of this intensity. If the random density takes only discrete values, which is a common situation in many physical problems where quantum mechanics introduces discrete states, it is shown that the thinning method can be applied without error. We study in particular the case of binary density and we present the kind of theoretical calculations that then become possible. The results of various experiments realized with data obtained by simulation show a fairly good agreement with the theoretical calculations.
\end{abstract}

Some key words. Point processes, lifetime, counting.

\section{Introduction}

Point processes (PP) play an important role in various domains of Science, Technology and Economics. In the one-dimensional case, which is the only one considered in the following pages, they are convenient to describe events that arrive randomly in time either at a microscopic scale (emission of photons or electrons) or at a macroscopic scale (traffic problems). There exists abundant literature for their presentation as, for example (Cox et al., 1980; Snyder et al., 1991; Lowen et al. 2005). Explicit calculations of mathematical properties of PPs are often very complicated to the effect that, in a number of cases,

\footnotetext{
${ }^{1}$ Unité mixte de recherche (UMR 8506) du Centre national de la recherche scientifique (CNRS), de l'École supérieure d'électricité (Suplec) et de l'Université de Paris-Sud 11 (UPS).
} 
the only possible approach for their study is by using simulation procedures, which is one of the main purposes of this paper.

They exist various papers or books devoted to simulation of Poisson processes. In the stationary case the problem is quite straightforward because the distances between successive points are IID positive random variables (RV) with an exponential distribution. The simulation of a stationary Poisson process is then reduced to the generation of a sequence of IID positive exponential RVs, which is a very simple problem.

The question becomes more difficult in the case on nonstationary (or nonhomogeneous) Poisson processes. A nonstationary Poisson process is entirely defined by its density $\lambda(t),(\lambda(t) \geq 0)$, sometimes also called intensity or rate function. It is often useful to introduce the cumulative rate function $\Lambda(t)=\int_{0}^{t} \lambda(\theta) d \theta$, which is the mean value of the number of points of the process in the interval $[0, t]$. If the inverse function $\Lambda^{-1}(s)$ of $\Lambda(t)$ can be calculated explicitly, this provides a simple simulation method because the nonstationary Poisson process can be obtained by an inversion procedure from a stationary Poisson process. The use of this method, however, is often impossible because of the lack of knowledge of $\Lambda^{-1}(s)$.

In this case, the most convenient method for the simulation of a nonstationary Poisson process is by using the thinning procedure. As said in (Devroye, 1986), p. 251, "the major work on simulation of nonhomogeneous Poisson processes is (Lewis, 1979)". Until recently various authors have presented some improvements concerning simulations of nonstationary Poisson processes, especially for applications in problems related to insurance and risks (Harrod et al., 2006, Burnecki et al., 2004) and also in biology (Shinomoto, 2001).

In this paper we are interested in simulation problems of doubly stochastic Poisson processes (DSPP). This expression refers to nonstationary Poisson processes in which the density $\lambda(t)$ becomes a positive random signal $\lambda(t, \omega)$, where $\omega$ stands for randomness. The first and most important use of such PPs was for Statistical Optics (Saleh, 1978; Lowen et al., 2005; Picinbono et al., 2010), which was the origin of various studies in optical communications and information theory, some of them being summarized in (Snyder et al., 1991). DSPPs, however, are not limited to physics, and also appear in numerous other domains such as, for example, management, economics, finance and traffic 
problems (Burnecki et al., 2004; Centanni et al., 2006; Lowen et al., 1995).

Since DSPPs are extensions of nonstationary Poisson processes, it seems natural to extend for their simulation the thinning method introduced in the original and basic paper (Lewis et al., 1979). This was explicitly announced in the last sentence of this paper saying "It is also possible to extend the method of thinning to simulation of doubly stochastic or conditioned Poisson processes. This will be discussed elsewhere." At least to our knowledge, this intention was never realized but realized some years late in (Ogata, 1981). Another attempt in this direction was presented in (Apanasovich et al., 1993), but the results are not convincing and the theoretical presentation is rather obscure. We shall below indicate other more recent approaches of this problem.

The paper is organized as follows. In Section 2 we recall the principles of the thinning method introduced in (Lewis et al., 1979) for the simulation of nonstationary Poisson processes. We indicate how this procedure can be extended for DSPPs and we present the problems appearing for this extension due to the fact that the density of a DSPP is random. In Section 3 we present a statistical model of random density for which these problems can be solved without approximation. This model is constructed with the help of a random process called random telegraph signal (RTS) widely used in many other areas of physics and signal processing. In Section 4 we calculate some statistical properties of the integral of the RTS that are useful for the following mathematical analysis. Various computer simulations confirm the validity of these calculations. In Section 5 we show that the model of random density with discrete values allows us to use the thinning method for the computer simulations of a DSPP and various experimental measurements on these simulations are presented in the last section. We show especially that measurements of moments of the random number of points in some time intervals or of PDFs of the lifetimes of such PPs yield results very similar to those obtained from the calculations.

\section{Principles and problems of the thinning method}

The principle of the thinning method is derived from the following result. Consider a Poisson stationary point process $\mathcal{P}_{P S}$ of density $\mu$ and let $\lambda(t)$ be 
a bounded nonnegative function satisfying $\lambda(t)<\mu$. Let $T_{i}$ be the random points (or time instants) of $\mathcal{P}_{P S}$ noted in increasing order $\left(T_{i}<T_{i+1}\right)$. To each $T_{i}$ we associate a thinning coefficient $R_{i}$ which is a Bernoulli RV taking only the values 0 or 1 . We assume that these RVs are independent of each other and also independent of $\mathcal{P}_{P S}$. Furthermore we suppose that, for a given realization of $\mathcal{P}_{P S}$ in which the $T_{i}$ s take the values $T_{i}=t_{i}$, the probability that $R_{i}=1$ is equal to $\lambda\left(t_{i}\right) / \mu$. Finally we suppose that if $R_{i}=0$ the point $t_{i}$ is erased and that it is not erased if $R_{i}=1$. The non erased points of $\mathcal{P}_{P S}$ constitute a new point process $\mathcal{P}$ and it results from all these assumptions that $\mathcal{P}$ is a nonstationary Poisson process of density $\lambda(t)$ (Lewis et al., 1979).

From this result there are various ways to obtain $\mathcal{P}$ from computer simulations. The simplest one is to adapt to this problem the techniques already used for the analysis of dead time effects in PPs (Picinbono, 2009) which also leads to a problem where an initial PP $\mathcal{P}_{I}$ is transformed into another one $\mathcal{P}$ by thinning. In this last case, however, the statistical properties of the coefficients $R_{i}$ are defined from the physical structure of the dead time effects, while in our problem they are defined from the previous assumptions.

Suppose now that $\mathcal{P}$ is a DSPP defined by a random density $\lambda(t, \omega)$. This means that for each value of $\omega, \mathcal{P}$ is a nonstationary Poisson process of density $\lambda(t, \omega)$. We can then apply the thinning method indicated above to generate a trajectory of a DSPP from that of a pure stationary Poisson process $\mathcal{P}_{P S}$ of density $\mu$. The validity of this procedure was shown by (Ogata, 1981).

Two problems however immediately appear. The first one comes from the principle of this method. Indeed we have seen that one of its basic assumptions is that the density $\lambda(t)$ of the PP $\mathcal{P}$ to be simulated must be bounded. When this density becomes random, $[\lambda(t, \omega)]$, it has in general no reason to be bounded, which makes impossible to satisfy the conditions of validity of the thinning procedure. For example if $\lambda(t, \omega)$ is the square of a normal signal, it is positive but not bounded. This is the reason why we shall study in the following statistical models of random density ensuring that it is bounded.

The second problem concerns the calculation of the coefficients $R_{i}$ used in the thinning procedure. We have seen above that the probability that $R_{i}=1$ is equal to $\lambda\left(t_{i}\right) / \mu$. For a given value of $\omega$, or for a given trajectory of the random density of the PP to be simulated, we must then calculate $\lambda\left(t_{i}, \omega\right)$. 
The instants $t_{i}$ are the points in the time axis of a realization of a stationary Poisson process $\mathcal{P}_{P S}$ of density $\mu$ and in simulation procedure they are obtained from a realization of a sequence of IID positive exponential RVs. Consider now the calculation of $\lambda\left(t_{i}, \omega\right)$. If the analytic expression of this function is known, which is often the case when $\lambda(t)$ is not random, this can easily be done. On the other hand when $\lambda(t)$ becomes random, the analytic expression of each trajectory is very rarely known and it is in general described only from some of its statistical properties.

In simulation experiments we must work with a density also obtained by simulation and this implies that in most of the cases this density is in practice only known at certain time instants $\tau_{i}$ coming from the simulation procedure. These instants have no relation with the $t_{i}$ s coming from the simulation of the $\mathrm{PP} \mathcal{P}_{P S}$. Then, starting from times $t_{i}$ we must estimate $\lambda\left(t_{i}, \omega\right)$, while we have at our disposal the values $\lambda\left(\tau_{i}, \omega\right)$. This requires the use of some approximations introducing a systematic bias in the procedure. Similar problems also appear with other simulations methods and some are discussed in (Giesecke et al., 2011). As seen below, this disadvantage completely disappears when using random densities taking only two possible values, which is a limit case of a piecewise-constant density used in (Harrod et al., 2006).

As we shall see later, it is possible to overcome without approximation the two previous difficulties of the thinning method for the simulation of DSPPs by using a binary density. Furthermore such a density is a rather good approximation of various physical situations. For example signals taking only two possible values are widely used in digital communications and information theory and in optical digital communications a point process with a binary intensity corresponds to various physical devices.

It is however interesting to note that DSPP are recently widely used in mathematical analysis of financial markets playing an essential role in the evolution of the world economic situation. A long list of papers appears in the reference list of (Giesecke, $2011 \mathrm{a}$ and b). As in statistical physics many studies require the use of simulation procedures and there are various attempts in order to simulate Poisson processes with random intensity. The two papers cited above present the most complete overview of the results obtained in this field. Looking at this literature, the first point that clearly appears is that discrete 
valued intensities are not at all appropriate in this field for the description of random intensities of PPs. The fluctuations of the financial parameters studied are more conveniently described by diffusion or Brownian motion processes. Furthermore the purpose of the simulation are rarely the properties of the simulated PP and more often some particular quantities especially interesting in financial problems such as loss or gain or evaluation of the risks. This is also the case of papers in the domain of insurance business. Even with this difference of perspective, the two problems appearing in the simulation of DSPPs and presented above are well noted and an attempt for their solution is introduced in the two papers of Giesecke cited in references. By using very abstract mathematical procedures of measure and martingale theory, Giesecke introduces a specific model of diffusion for the random intensity. It becomes then possible to use the thinning method for the simulation of DSPPs even for unbounded density, which is the case of diffusion processes, and to suppress the bias in the estimation of the sampling of the random intensity. The algorithms deduced from the theoretical analysis are appropriate for the domain of the paper but seem without interest in statistical physics, in such a way that the is almost no overlapping between these methods and those discussed below.

\section{Doubly stochastic Poisson processes with binary density}

Let us begin by showing the origin of the theoretical difficulties that appear in almost all the calculations with DSPPs, which justifies the necessity of a simulation approach. Consider for example the case of counting analysis which is one of the most common ways to study PPs. Let $N$ be the random number of points of a $\mathrm{PP}$ in some interval which can arbitrarily be $[0, T]$. If the $\mathrm{PP}$ is a Poisson process of density $\lambda(t)$, the probability $p_{n}$ that $N=n$, where $n$ is an arbitrary nonnegative integer, is $p_{n}=\exp (-m) m^{n} / n$ ! where $m=\int_{0}^{T} \lambda(\theta) d \theta$.

In the case of a DSPP, $m$ becomes random, because of the randomness of $\lambda(t, \omega)$, in such a way that

$$
p_{n}=\mathrm{E}\left[\exp (-M) \frac{M^{n}}{n !}\right] \quad \text { with } \quad M=\int_{0}^{T} \lambda(\theta, \omega) d \theta
$$

where $\mathrm{E}$ means the expected value with respect to $\omega$ specifying the randomness of the density of the PP. As said on p. 171 of (Saleh, 1978), where this problem 
is discussed, "To find the statistics of an integral over a stochastic process is, in general, a formidable task". This is effectively the origin of the difficulty of the calculations of the probabilities $p_{n}$ because of the lack of knowledge of the statistics of the random variable $M$ appearing in (1).

Various attempts have been made to solve this problem. However the number of cases where an explicit solution can be found remains very limited. The first approach in the domain of statistical optics was presented in (Bédard, 1966) and an overview of some other cases is given in (Saleh, 1978). This requires in general a Karhunen Loeve expansion for which it is necessary to solve an integral equation and explicit analytical expressions are rarely obtained. Another simplification is often used in optics in the so-called long coherence time case. This means that the variations of $\lambda(t, \omega)$ are very small in intervals of duration of the order of $T$ in such a way that in (1) the integral over $[0, T]$ can be replaced by $\lambda(0, \omega) T$, which in a number of cases makes it possible to calculate of the probabilities $p_{n}$.

Let us now present an example of random density in which the previous difficulties concerning the conditions of simulation by thinning or the mathematical calculations disappear. This density is defined by a general expression of the form

$$
\lambda(t, \omega)=\lambda_{0}[1+\alpha B(t, \omega)]
$$

where $B(t, \omega)$ is a random perfect clipped signal taking only the values \pm 1 , the changes of signs appearing at the points of a $\mathrm{PP} \mathcal{P}_{B}$ assumed to be stationary. It is first clear that, whatever the nature of $\mathcal{P}_{B}$, if $|\alpha|<1$, then the random density $\lambda(t, \omega)$ is positive and bounded, which ensures that the first condition of validity of the thinning method is satisfied.

Let us show that this is also the case for the second condition. Let $S_{i}$ denote the ordered sequence of the random points of $\mathcal{P}_{B}$ and $s_{i}$ a particular realization of this sequence. It results from the definition of $B(t)$ that in the interval $s_{i}<t<s_{i+1}$ it remains constant and equal to $B\left(s_{i}^{+}\right)$, the value of $B(t)$ obtained immediately after the change of sign appearing at $s_{i}$. As a result, if $B\left(s_{0}^{+}\right)$is known we have $B(t)=B\left(s_{0}^{+}\right)(-1)^{i}$ for any $t$ satisfying $s_{i}<t<s_{i+1}$.

From an algorithmic point of view we see that in order to calculate $B(t)$ we must associate to any value of $t$ the integer $i(t)$ such that $s_{i(t)}<t<s_{[i(t)+1]}$. Applying the previous relation we obtain $B(t)=B\left(s_{0}^{+}\right)(-1)^{i(t)}$, valid for any 
$t$. There are various ways to obtain $i(t)$, the simplest one coming from the fact that $i(t)$ is the only integer such that the product $\left[t-s_{i(t)}\right]\left[t-s_{[i(t)+1]}\right]$ is negative. This exact knowledge of the values of $B(t)$ for any value of $t$ allows one to use the thinning algorithm, according to the previous discussion.

It remains to show that in some cases this model introduces the possibility to make analytical calculations which in general are almost impossible. This is especially the case when the $\operatorname{PP} \mathcal{P}_{B}$ is a stationary Poisson process defined by its density $\nu$.

In this specific case the signal $B(t)$ introduced above becomes the random telegraph signal (RTS), widely used in random signal theory. It is introduced on p. 334 of (Picinbono, 1993) and studied as a particular case of ordered signals in (Picinbono, 1999). The main property is that all its moments of order higher than two have a simple mathematical expression, in such a way that, as shown in the next section, the calculation of various statistical properties of the integral appearing in (2) becomes possible. It is worth pointing out that the RTS is not only a simple mathematical extension of Poisson processes, but also a rather good statistical model to represent physical phenomena in which a system makes random transitions between two states, which is a very common situation (Efros et al., 1997). It is also widely used in binary communications, which is the origin of its name, and densities such as $(2)$, where $B(t)$ is a RTS, can be used in a description of binary optical communications.

\section{Statistics of the integral of the RTS}

Let $B(t)$ be the RTS of a stationary Poisson process $\mathcal{P}_{B}$ of density $\nu$ and $Z$ the integral

$$
Z(t, T) \triangleq \int_{t}^{t+T} B(\theta) d \theta
$$

Because of the stationarity of $\mathcal{P}_{B}$ the statistical properties of $Z$ do not depend on $t$. The moment of order $k$ of $Z$ is given by

$$
M_{Z_{k}}=\int \ldots \int_{D} \mathrm{E}\left[B\left(\theta_{1}\right) B\left(\theta_{2}\right) \ldots B\left(\theta_{k}\right)\right] d \theta_{1} d \theta_{2} \ldots d \theta_{k},
$$

where $D$ is a $k$-dimensional cube of edge length $T$. The expression of the high order moments of $B(t)$ appearing in (4) is well known (see Picinbono, 1999). The moments of odd order are zero and for even order we have

$$
\mathrm{E}\left[B\left(\theta_{1}\right) B\left(\theta_{2}\right) \ldots B\left(\theta_{2 k}\right)\right]=\exp \left[-2 \nu\left(\bar{\theta}_{2}-\bar{\theta}_{1}+\bar{\theta}_{4}-\bar{\theta}_{3}+\ldots+\bar{\theta}_{2 k}-\bar{\theta}_{2 k-1}\right)\right],
$$


where $\left\{\bar{\theta}_{i}\right\}$ is the ordered permutation of $\left\{\theta_{i}\right\}$, satisfying $\bar{\theta}_{j}<\bar{\theta}_{j+1}, \forall j, 1 \leq$ $j<2 k-1$. After what are sometimes rather complicated calculations it is possible to deduce from (5) the values of the moments $M_{Z_{k}}$ defined by (4). For example the moments of order 2 and 4 are given by

$$
M_{Z_{2}}=(1 / \nu)\left[T-(1 / 2 \nu)\left(1-\mathrm{e}^{-2 \nu T}\right)\right]
$$

and

$$
M_{Z_{4}}=\left(3 / 2 \nu^{4}\right)\left[3-4 \nu T+2(\nu T)^{2}-(3+2 \nu T) \mathrm{e}^{-2 \nu T}\right] .
$$

Let us now see how the integral (3) can be calculated in a computer simulation experiment. Let $\xi_{i}$ be the distances between successive points in the Poisson process $\mathcal{P}_{B}$. As indicated above the instants $s_{i}$ of this PP are then defined by the recursion $s_{i}=s_{i-1}+\xi_{i}$. It results from the structure of the RTS that for any arbitrary positive integer $N$

$$
Z\left(s_{i}, s_{i+N}-s_{i}\right)=\int_{s_{i}}^{s_{i+N}} B(\theta) d \theta=B\left(s_{i}^{+}\right) \sum_{k=1}^{N}(-1)^{k-1} \xi_{i+k},
$$

where $B\left(s_{i}^{+}\right)$is defined above. Then the integral of the RTS over an interval between two points of $\mathcal{P}_{B}$ is reduced to a sum of $N$ terms, where $N-1$ is the number of points of the PP between $s_{i}$ and $s_{i+N}$. Consider now the calculation of $Z\left(s_{i}, T\right)$. It is clear that $s_{i}+T$ is almost surely not a point of $\mathcal{P}_{B}$. There exist however simple algorithms that make it possible to calculate the number $N_{i}$ of points of $\mathcal{P}_{B}$ appearing in the interval $\left[s_{i}, s_{i}+T\right]$ and they are deduced from the inequalities $s_{i+N_{i}}<s_{i}+T<s_{i+N_{i}+1}$. As a result we have

$$
Z\left(s_{i}, T\right)=Z\left(s_{i}, s_{i+N_{i}}-s_{i}\right)+\int_{s_{i+N_{i}}}^{s_{i}+T} B(\theta) d \theta .
$$

Since $s_{i+N_{i}}$ is the last point of $\mathcal{P}_{B}$ before $s_{i}+T$, there is no change of sign of $B(\theta)$ in the interval defining the last integral of (9). Then it is equal to $B\left(s_{s_{i}^{+}+N_{i}}\right) \Delta \theta$, where $\Delta \theta=s_{i}+T-s_{i+N_{i}}$. As the $\xi_{i}$ s are the intervals between successive points of $\mathcal{P}_{B}$ defined recursively by $s_{i+1}=s_{i}+\xi_{i+1}$, we have $s_{i+N_{i}}=s_{i}+\xi_{i+1}+\ldots+\xi_{i+N_{i}}$, which allows us to calculate $Z\left(s_{i}, T\right)$ uniquely from the sequence of $M$ values $\xi_{i}$, which are $M$ realizations of IID positive exponential RVS. This the starting point of the statistical analysis that follows in which $M$ is of the order of $10^{6}$.

In Table 1 we present results concerning the first four moments of $Z(t, T)$. Their theoretical values are given above by (6) and (7) and furthermore $M_{Z_{1}}=$ 
$M_{Z_{3}}=0$. These moments are calculated and measured for two values of $T$ and several values of the density $\nu$ of $\mathcal{P}_{B}$ The theoretical and experimental results are noted $M_{Z_{k}}$ and $\hat{M}_{Z_{k}}$ respectively. This table shows a very good agreement between the theoretical and experimental values. The precision of the method decreases when $\nu T$ increases which comes from the structure of the algorithm of simulation. Indeed, as said above, for each $i$ we have to calculate the numbers $N_{i}$ defined above which are RVs of mean value $\nu T$. In order to reduce the duration of the calculation the $N_{i}$ greater than a certain limit are deleted. This clearly introduces a bias in the measurements which increases with $\nu T$. It can obviously be reduced but at the price of longer calculations.

Table 1. Moments of the Integral of the RTS.

\begin{tabular}{|c|c|c|c|c|c|c|c|c|c|}
\hline & $\nu$ & $M_{Z_{1}}$ & $\hat{M}_{Z_{1}}$ & $M_{Z_{2}}$ & $\hat{M}_{Z_{2}}$ & $M_{Z_{3}}$ & $\hat{M}_{Z_{3}}$ & $M_{Z_{4}}$ & $\hat{M}_{Z_{4}}$ \\
\hline \multicolumn{10}{|l|}{$T=1$} \\
\hline & 0.5 & 0 & 0.0004 & 0.7358 & 0.7356 & 0 & 0.0005 & 0.6836 & 0.6836 \\
\hline & 1.0 & 0 & -0.0002 & 0.5677 & 0.5678 & 0 & -0.0002 & 0.4850 & 0.4852 \\
\hline & 1.5 & 0 & -0.0003 & 0.4555 & 0.4553 & 0 & -0.0003 & 0.3559 & 0.3558 \\
\hline & 2.0 & 0 & 0.0003 & 0.3773 & 0.3775 & 0 & 0.0002 & 0.2692 & 0.2693 \\
\hline & 2.5 & 0 & -0.0006 & 0.3205 & 0.3206 & 0 & -0.0004 & 0.2091 & 0.2093 \\
\hline & 3.0 & 0 & -0.0004 & 0.2779 & 0.2783 & 0 & -0.0002 & 0.1663 & 0.1668 \\
\hline \multicolumn{10}{|l|}{$T=2$} \\
\hline & 0.1000 & 0 & 0.0001 & 3.5160 & 3.5158 & 0 & -0.0016 & 13.6777 & 13.6771 \\
\hline & 0.3000 & 0 & -0.0002 & 2.7844 & 2.7845 & 0 & -0.0007 & 10.1823 & 10.1814 \\
\hline & 0.5000 & 0 & 0.0012 & 2.2707 & 2.2701 & 0 & 0.0033 & 7.7598 & 7.7594 \\
\hline & 1.0000 & 0 & -0.0016 & 1.5092 & 1.5089 & 0 & -0.0009 & 4.3077 & 4.3086 \\
\hline & 1.5000 & 0 & -0.0025 & 1.1117 & 1.1130 & 0 & -0.0048 & 2.6601 & 2.6689 \\
\hline
\end{tabular}

Let us now present some results concerning measurements of probability density functions (PDF). Our purpose is to determine by computer simulated experiments the PDF of the RV $Z(t, T)$. This PDF does not depend on $t$ because of the assumption that $\mathcal{P}_{B}$ is stationary. We can a priori expect that the distribution of $Z$ contains a discrete part. Indeed if there is no point of $\mathcal{P}_{B}$ in the interval $[t, t+T]$ the possible values of the RV $Z$ are $\pm T$ because there is no change of sign of the function $B(\theta)$ in the interval of integration. Since $\mathcal{P}_{B}$ is a Poisson process, the probability that the interval $[t, t+T]$ does not contain points of $\mathcal{P}_{B}$ is $\exp (-\nu T)$. Then we have $\operatorname{Pr}(Z= \pm T)=\exp (-\nu T)$. This probability can also be obtained from computer simulation by using the same kind of experimental approach as previously and the results appear in Table 2. For two values of $T$ we present the theoretical value of $\operatorname{Pr}(Z= \pm T)$ and the results of four independent experiments. The agreement between theory and experiment is quite good. 
On the other hand there is no simple theoretical expression of the continuous part of the PDF $p_{Z}(z)$ of $Z$ given by (3). This PDF can be measured and the results appear on Fig. 1 obtained for $\nu=T=1$. The experimental values of the PDF are located with a rather good precision on the curve $-0.1 z^{2}+0.54$, which does not have a simple explanation.

Table 2. Probability that $Z$ of (3) is equal to $\pm T$

\begin{tabular}{ccc}
\hline$T=1$ & $T=2$ \\
\hline Th. & 0.3679 & 0.1353 \\
\hline Exp. & 0.3676 & 0.1353 \\
\hline & 0.3682 & 0.1352 \\
\hline & 0.3678 & 0.1358 \\
\hline & 0.3681 & 0.1355 \\
\hline
\end{tabular}

\section{Algorithms for the simulation of a DSPP with binary density}

Following the definitions introduced in Section 3 we shall now present the sequences of algorithms used for the simulation of a DSPP with a binary density given by $(2)$ where $B(t)$ is the RTS defined from a stationary Poisson process $\mathcal{P}_{B}$.

The first step is to generate a sequence of $N$ ordered points $t_{i}$ of the stationary Poisson process $P_{P S}$ of density $\mu$. This is a straightforward standard task. Indeed the intervals between the successive points of this process are IID positive RVs with the exponential distribution $\mu \exp (-\mu x)$ easily obtained from various computer programs.

The second step is quite similar and yields the sequence of ordered points $s_{j}$ of another stationary Poisson process $P_{B}$ of density $\nu$. These points are the instants at which the RTS $B(t)$ changes its value from \pm 1 to $\mp 1$.

The third step is to associate to each $t_{i}$ of $P_{P S}$ the point $s_{j(i)}$ of $P_{B}$ such that the product $\left(t_{i}-s_{j(i)}\right)\left(t_{i}-s_{j(i)+1}\right)$ is negative. This can be done in two ways. In the first, indicated above, for each $t_{i}$ we calculate the $N$ products $\left(t_{i}-s_{j}\right)\left(t_{i}-\right.$ $\left.s_{j+1}\right)$ and $j(i)$ is the only value of $j$ for which this product is negative. This procedure however requires very long calculation times because there are of the order of $N^{2}$ products to calculate which yields in our experiments an order of $10^{12}$ multiplications. With standard computers this can require more than several hundred hours of calculations. It is then more appropriate to introduce 
a recursive procedure which is much less time consuming. Its principle is as follows.

Suppose that $s_{j(i)}$ is known and consider the difference $d_{n}(i+1)=t_{i+1}-$ $s_{j(i)+n}$. It is clear that $j(i+1)=j(i)+n$ if and only if $d_{n}(i+1)>0$ and $d_{n+1}(i+1)<0$. This yields that

$$
j(i+1)=j(i)+\sum_{n=1}^{\infty} n F_{n}(i)
$$

with

$$
F_{n}(i)=u\left[d_{n}(i+1)\right] v\left[d_{n+1}(i+1)\right]
$$

where $u(x)$ is the unit step function equal to 1 if $x>0$ and to -1 otherwise, and $v(x)=u(-x)$. The advantage of (10) seems weak, because the presence of a series of an infinite number of terms. It appears however that this number is in practice very small. In particular when the densities of the PPs $P_{P S}$ and $P_{B}$ satisfy $\nu<<\mu$, which is a very common situation, a short calculation shows that there is practically only one term corresponding to $n=1$ in the series of (10), and for each set of experiments we have introduced a procedure ensuring that replacing the series by a sum of a finite number of terms does not introduce an error in the calculation of (10).

The fourth step is to calculate the value of the thinning coefficient $R_{i}$ defined in the first paragraph of Section 2. It is a Bernoulli RV and the probability that the point $t_{i}$ be not erased, event corresponding to $R_{i}=1$, is $\lambda\left(t_{i}\right) / \mu$. This requires the knowledge of $\lambda\left(t_{i}\right)$. It is the value of the density (2) for $t=t_{i}$ which is known when $B\left(t_{i}\right)$ is known. For this we use the expression $B(t)=B\left(s_{0}^{+}\right)(-1)^{i(t)}$ indicated in Section 3. It results from the definition of $s_{j(i)}$ that $B\left(t_{i}\right)=B\left(s_{0}^{+}\right)(-1)^{j(i)}$. Using the values of $B\left(t_{i}\right)$, and then of $\lambda\left(t_{i}\right)$, associated with each point $t_{i}$ of $\mathcal{P}_{P S}$, we can apply the thinning procedure and the non erased points from this procedure constitute a trajectory of the simulated DSPP to be used for the following experiments.

\section{$6 \quad$ Presentation of experimental results}

Since it is very rarely possible to carry out theoretical calculations of statistical properties of DSPPs, our first task is to introduce computer experiments in which the results can be compared to those obtained from a mathematical 
analysis. This is especially the case for the study of the moments of the random number of points counted in a given time interval (counting experiments). As indicated in various papers, it is necessary, except for Poisson processes, to make a clear distinction between relaxed and triggered counting experiments. In the relaxed case the beginnings of the counting time intervals have no relation with the PP analyzed, while in the triggered case they depend on this PP, the simplest example being the one where the counting intervals are open by a point of the PP. Since the theoretical analysis is much more complicated for the triggered case, we shall only present results concerning relaxed counting experiments, which are also in general simpler to realize than the triggered ones.

Let us note $N$ the number of random points of a stationary DSPP in an interval $[t, t+T]$. It is a positive $\mathrm{RV}$ and the main purpose of counting experiments is to determine some properties of its probability distribution. Its theoretical calculation is in general very difficult, even for the particular model of random density introduced in this paper. On the other hand, we shall now verify that the results obtained in the previous sections make the theoretical analysis of the moments of the RV $N$ much simpler.

The first four moments of a Poisson RV $N$ of mean $m$ are given by

$$
\begin{aligned}
\mathrm{E}(N) & =m, \\
\mathrm{E}\left(N^{2}\right) & =m+m^{2}, \\
\mathrm{E}\left(N^{3}\right) & =m+3 m^{2}+m^{3}, \\
\mathrm{E}\left(N^{4}\right) & =m+7 m^{2}+6 m^{3}+m^{4} .
\end{aligned}
$$

If $N$ is now the number of points of a DSPP recorded in the arbitrary time interval introduced above (relaxed counting), its moments are simply obtained by replacing the quantities $m^{k}$ of these relations by $\mathrm{E}\left(M^{k}\right)$, where $M$ is given by (1) in which 0 and $T$ are replaced by $t$ and $t+T$ respectively. For the calculation of these expected values we start from (2) which introduces values of the integral of the RTS calculated above. By using the notations previously introduced we obtain

$$
\begin{aligned}
\mathrm{E}(M) & =\lambda_{0} T \\
\mathrm{E}\left(M^{2}\right) & =\lambda_{0}^{2}\left[T^{2}+\alpha^{2} M_{Z_{2}}\right]
\end{aligned}
$$




$$
\begin{aligned}
& \mathrm{E}\left(M^{3}\right)=\lambda_{0}^{3}\left[T^{3}+3 \alpha^{2} T M_{Z_{2}}\right] \\
& \mathrm{E}\left(M^{4}\right)=\lambda_{0}^{4}\left[T^{4}+6 \alpha^{2} T^{2} M_{Z_{2}}+\alpha^{4} M_{Z_{4}}\right]
\end{aligned}
$$

where $M_{Z_{2}}$ and $M_{Z_{4}}$ are given by (6) and (7) respectively. Replacing $m^{k}$ by $\mathrm{E}\left(M^{k}\right)$ in Eqs (12) to (15) yields the theoretical values $m_{k}=\mathrm{E}\left(N^{k}\right)$ of the moments of order $k$ of the random number of points of the DSPP considered. These moments can also be measured by a procedure described in (Picinbono, 2012) applied to a computer simulation of this PP obtained by the procedure introduced above. The experiment is realized with the parameters $\mu=1$, $\lambda_{0}=1 / 2$ and $T=2$ in such a way that the mean value of the random number of points is 1 . Furthermore various values of the parameter $\alpha$ defining the variance of the fluctuations of the random density of the $\mathrm{PP}$ are taken in consideration. For $\alpha=0$ the DSPP becomes a pure Poisson process. The results appear in Table 3.

Table 3. First Four Moments of the Number of Points

\begin{tabular}{ccccc}
\hline & $m_{1}$ & $m_{2}$ & $m_{3}$ & $m_{4}$ \\
\hline$\alpha=0$ & & & & \\
\hline Th. & 1 & 2 & 5 & 15 \\
\hline Exp. & 0.99 & 1.99 & 4.99 & 15.04 \\
\hline$\alpha=0.5$ & & & & \\
\hline Th. & 1.0000 & 2.1948 & 6.1687 & 21.0844 \\
\hline Exp. & 1.001 & 2.196 & 6.168 & 21.083 \\
\hline$\alpha=0.8$ & & & & \\
\hline Th. & 1.0000 & 2.4987 & 7.9919 & 30.7596 \\
\hline Exp. & 1.004 & 2.504 & 7.995 & 30.735 \\
\hline$\alpha=0.9$ & & & & \\
\hline Th. & 1.0000 & 2.6311 & 8.7867 & 35.0470 \\
\hline Exp. & 1.007 & 2.645 & 8.809 & 35.036 \\
\hline
\end{tabular}

The first two lines correspond to the value $\alpha=0$, which means that the DSPP is a Poisson process. The first four moments are 1, 2, 5, and 15. The experimental values obtained are almost the same. For the other values taken by the parameter $\alpha$ there is also quite a good correspondence between the theoretical and experimental values of the moments. This means that the moment analysis of the relaxed number of points of the PP in a time interval correctly exhibits the properties of a DSPP defined by the random density (2).

By using the procedure described in (Picinbono, 2012) it is also possible to measure the PDFs of the time intervals between successive points (lifetimes). 
Unfortunately, even for this particular case of DSPP the theoretical calculations of these PDFs are almost impossible and initially we shall only present the results of computer experiments. They appear in Fig.2. In this figure the points represent experimental measurements of the PDFs of the lifetimes obtained for two values of $\alpha$. For $\alpha=0$ the DSPP becomes a Poisson Process, as already verified in other experiments carried out previously. It results from (2) that since $\lambda_{0}=1 / 2$ and since the mean value of $B(t)$ is zero, then the density of the Poisson process obtained by this particular mode of thinning is also equal to $1 / 2$, which implies that the PDF of the lifetime is exponential with the density $p(x)=0.5 \exp (-0.5 x)$. This clearly appears on the figure where the points are the results of experimental measurements while the continuous curve corresponds to this theoretical DDP. On the other hand, as indicated above, there is no theoretical result concerning the PDF of the lifetime when $\alpha \neq 0$ and the figure presents experimental results obtained for a DSPP defined by $\alpha=0.9$. The only fact that exhibits this PDF is that there are two kinds of exponential behaviour corresponding approximately to the two values taken by the random density $\lambda(t)$ given by (2). We shall now conduct a more precise analysis of this point in a case where some calculations become possible.

This case, already introduced above, is often used in the interpretation of experimental results of statistical optics and corresponds to the so-called long coherence time approximation. This means that the fluctuations of $B(t)$ in $(2)$ are sufficiently slow so that in integrals such as $(3), B(t)$ remains constant and becomes a RV, which greatly simplifies the calculation of the statistical properties of this integral, which, without this assumption, is the major difficulty of the theoretical analysis. Looking at (2) we see that this approximation can be expressed in terms of the densities $\mu$ and $\nu$ of the PPs $\mathcal{P}_{P S}$ and $\mathcal{P}_{B}$ respectively by the relation $\mu>>\nu$. In the previous experiments these values were $\mu=1$ and $\nu=0.2$, which do not satisfy this inequality. The last experiments of this paper, on the other hand, are realized with the values $\mu=1$ and $\nu=10^{-3}$, which does correspond much better to the previous inequality. From this approximation we can deduce that the random density is now given by (2) where $B(t)$ is simply a RV taking the values \pm 1 with the same probabilities $1 / 2$. By using this approximation we can use a method introduced on p. 345 of (Picinbono, 1993) in order to calculate the PDF of two distinct time intervals 
appearing in the study of PPs. The residual lifetime is the random distance between an arbitrary point $A$ and the first point of the process posterior to $A$, while the lifetime or order one is the distance between two successive points of the PP. In general, it is almost impossible to calculate the PDFs of these RVs noted $p_{R L}(x)$ and $p_{L}(x)$ respectively. But under the assumption introduced just above and by using the methods introduced in (Picinbono, 1993) we obtain

$$
\begin{gathered}
p_{R L}(x)=(1 / 2)\left[\lambda_{1} \exp \left(-\lambda_{1} x\right)+\lambda_{2} \exp \left(-\lambda_{2} x\right)\right], \\
p_{L}(x)=\lambda_{1}^{2} \exp \left(-\lambda_{1} x\right)+\lambda_{2}^{2} \exp \left(-\lambda_{2} x\right),
\end{gathered}
$$

where

$$
\lambda_{1}=\lambda_{0}(1+\alpha), \lambda_{2}=\lambda_{0}(1-\alpha)
$$

The results of experimental measurements of the PDFs of simulated data are presented in Fig. 3. For these experiments we have used $510^{6}$ samples of exponential RVs for the generation of the $\mathrm{PP} \mathcal{P}_{P S}$ of density $\mu=1$. The density $\nu$ of the Poisson process generating the RTS $B(t)$ was $10^{-3}$ and finally $\alpha=0.9$.

The theoretical values give by (20) and (21) are represented by continuous curves while the results of PDF measurements appear as points in the figure. The agreement between the theoretical analysis and experimental results is rather good. There is a small difference between them and we have verified that it results from the insufficient approximation of the calculation. The results are better if we take $\nu<10^{-3}$, but the duration of the experiment also increases and obtaining greater precision would require more memory than we can obtain in our experiment. Nevertheless the main point that clearly appears from this experiment is the difference between the statistics of the lifetime and the residual lifetime, as predicted by the theoretical analysis.

\section{References}

Apanasovich, V., Novikov, E. (1993). Computer simulation of photoevents. Jour. Modern Optics, 40:213-218.

Bédard G. (1966). Photon counting statistics of Gaussian light . Phys. Rev., 151:1038-1039. Burnecki, K., Hardle, W., Weron, R. (2004). Simulation of risk Processes, in Encyclopedia of Actuarial Science, J. L. Teugels, B. Sundt Eds., Wiley, Chichester, vol. 3:1564-1570. accessible at 
http://www.im.pwr.wroc.pl/ hugo/publ/HSC_03_4_BurneckiHaerdleRWeron.pdf

Centanni, S., Minozzo, M. (2006). A Monte Carlo approach to filtering for a class of marked doubly stochastic Poisson processes, Journal of the Amer. Statist. Association, vol 101, No. 476, Theory and Methods:1582-1597.

Cox, D., Isham, V. (1980). Point Processes. London: Chapman and Hall.

Devroye, L. (1986). Non-Uniform Random Variate Generation, New-York: Springer-Verlag. accessible at http://cg.scs.carleton.ca/ luc/rnbookindex.html

Efros, Al., Rosen, M. (1997). Random telegraph signal in the photoluminescence intensity of a single quantum dot, Phys. Rev. Lett., vol 78: 1110-1113.

Giesecke, K., Smelov, D., (2011,a). Exact sampling of jump-diffusions. Accepted in Operations Research, accessible at http://www.stanford.edu/dept/MSandE/cgi-bin/people/faculty/giesecke/pdfs/exact.pdf

Giesecke, K., Kim, B., Zhu, S., (2011,b). Monte Carlo algorithms for default timing problems. Management Science, accessible at http://www.stanford.edu/dept/MSandE/cgi-bin/people/faculty/giesecke/pdfs/fast.pdf

Harrod, S., and Kelton, W. (2006). Numerical methods for realizing nonstationary Poisson processes with piecewise-constant instantaneous-rate functions, Simulation, 82-3: 147-157.

Lewis, P. A. W., Shedler, S. (1979). Simulation of nonhomogeneous Poisson processes by thinning, Naval Research Logistics Quarterly, vol 26 (3): 403-413.

Lowen, S., Teich, M. (1995). Estimation and simulation of fractal stochastic point processes. Fractals, vol 3, No. 1: 183-210.

Lowen, S., Teich, M. (2005). Fractal-Based Point Processes, Hoboken (N. J. ): Wiley.

Ogata, Y. (1981), On Lewis simulation method for point processes, IEEE Trans. Inform. Theory, IT-27: 23-31.

Picinbono, B. (1993). Random Signals and Systems, Englewood Cliffs, NJ: Prentice-Hall.

Picinbono, B. (1999). Polyspectra of ordered signals. IEEE Trans. Inf. Theory, 45: 22392252 .

Picinbono, B. (2009). Output dead-time in point processes. Communications in StatisticsSimulation and Computation, 8: 2198-2213.

Picinbono, B. (2012). Algorithms for point processes analysis. Communications in Statistics - Simulation and Computation, to be published in November 2012.

Picinbono, B., Bendjaballah, C. (2010). Some properties of point processes in statistical optics, Physical Review, vol A 82: 063811, 1-8.

Saleh, B. (1978). Photoelectron Statistics, Berlin : Springer.

Saleh, B., Teich, M. (1991). Fundamentals of Photonics. Hoboken (N. J. ): Wiley.

Shinomoto, S., Tsubo, Y (2001). Modeling spiking behavior of neurons with time-dependent Poisson processes. Physical Review, vol E 64: 041910, 1-13.

Snyder, D., Miller M. (1991). Random Point Processes in Time and Space. New York: Springer-Verlag. 


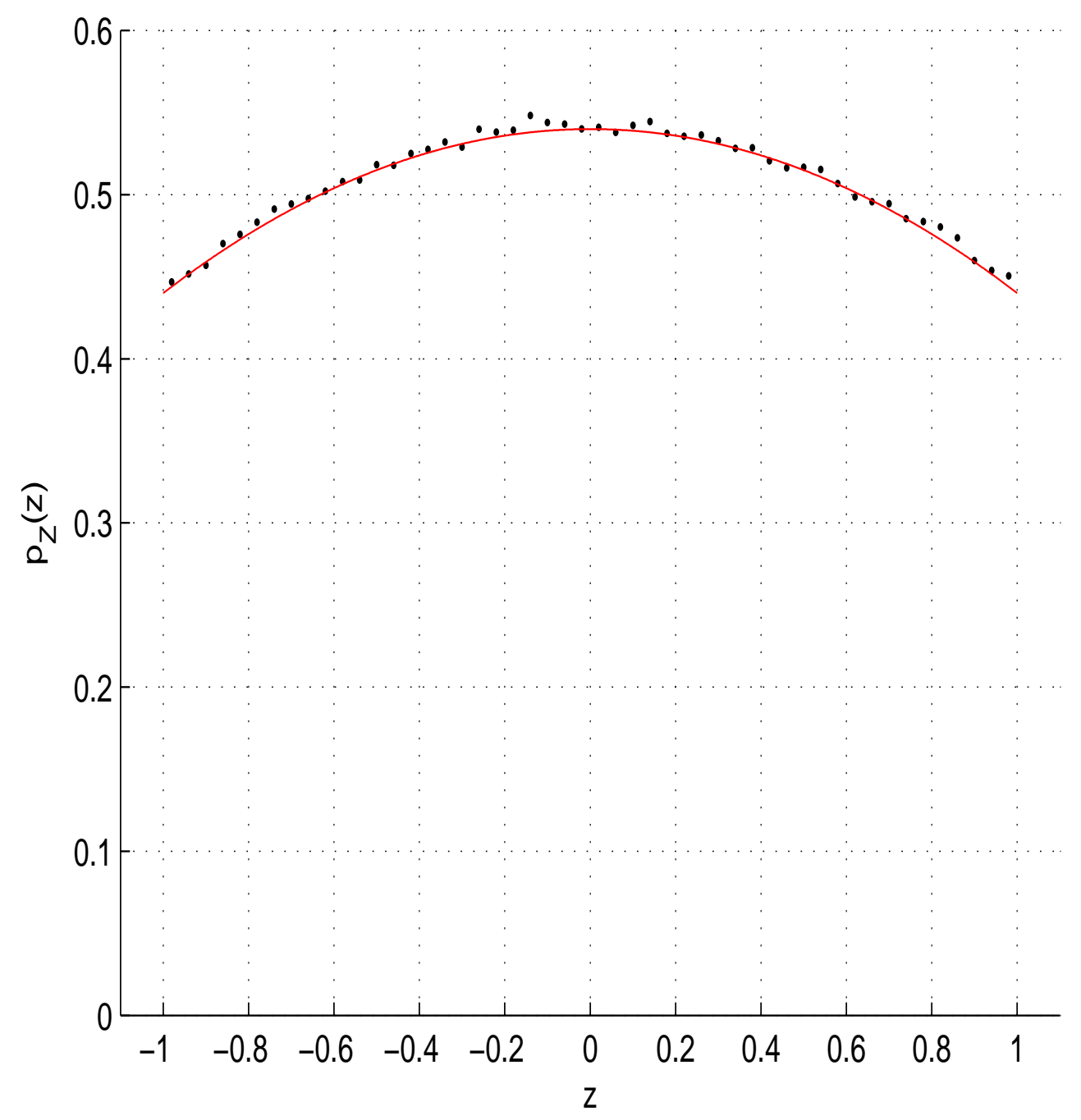

Figure 1: Probability density functions $p_{z}(z)$. Points: experiment, continuous curves: $-0.1 z^{2}+0.54$. 


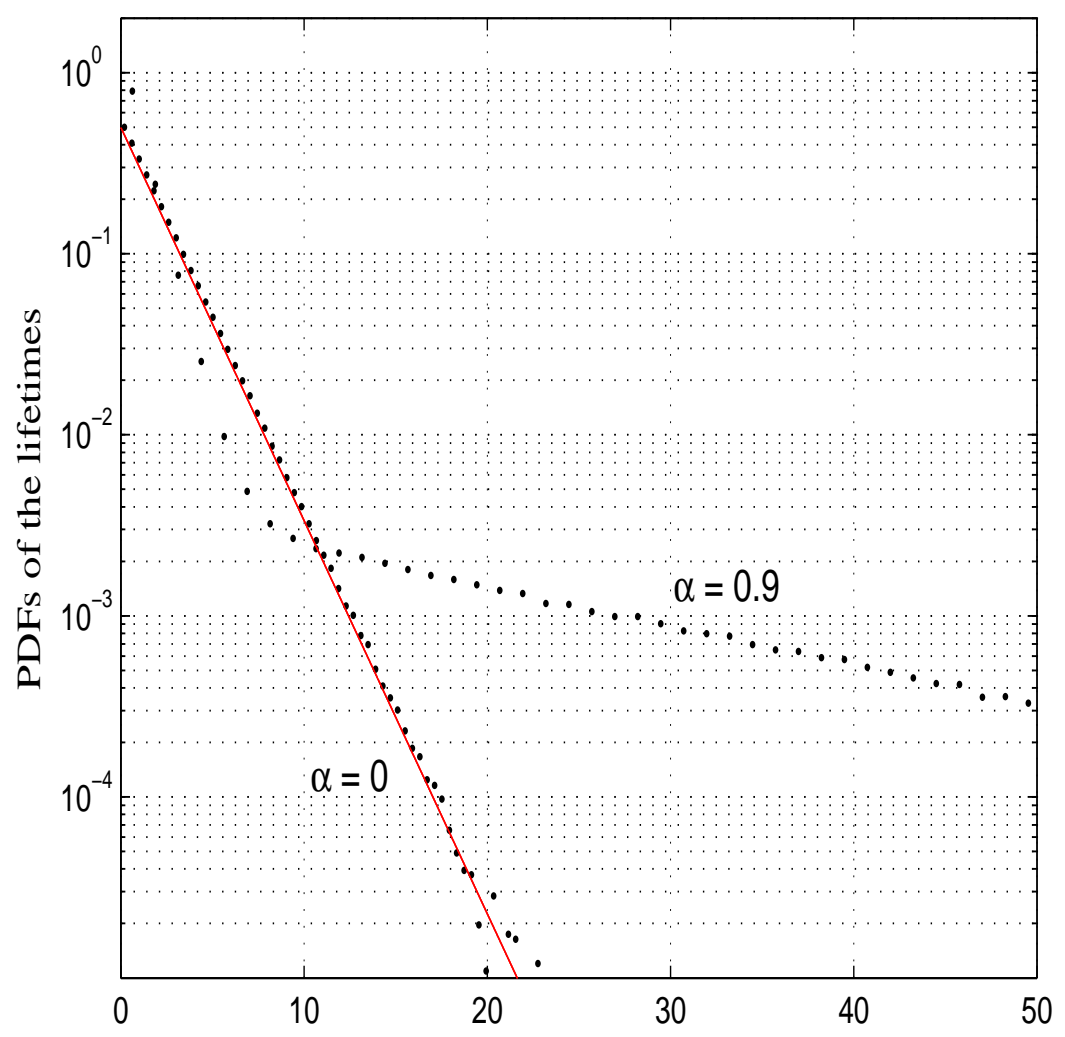

Figure 2: Probability density functions $p(x)$ of lifetimes of a DSPP for various values of $a$. Points: experiment, continuous curves: theory. 


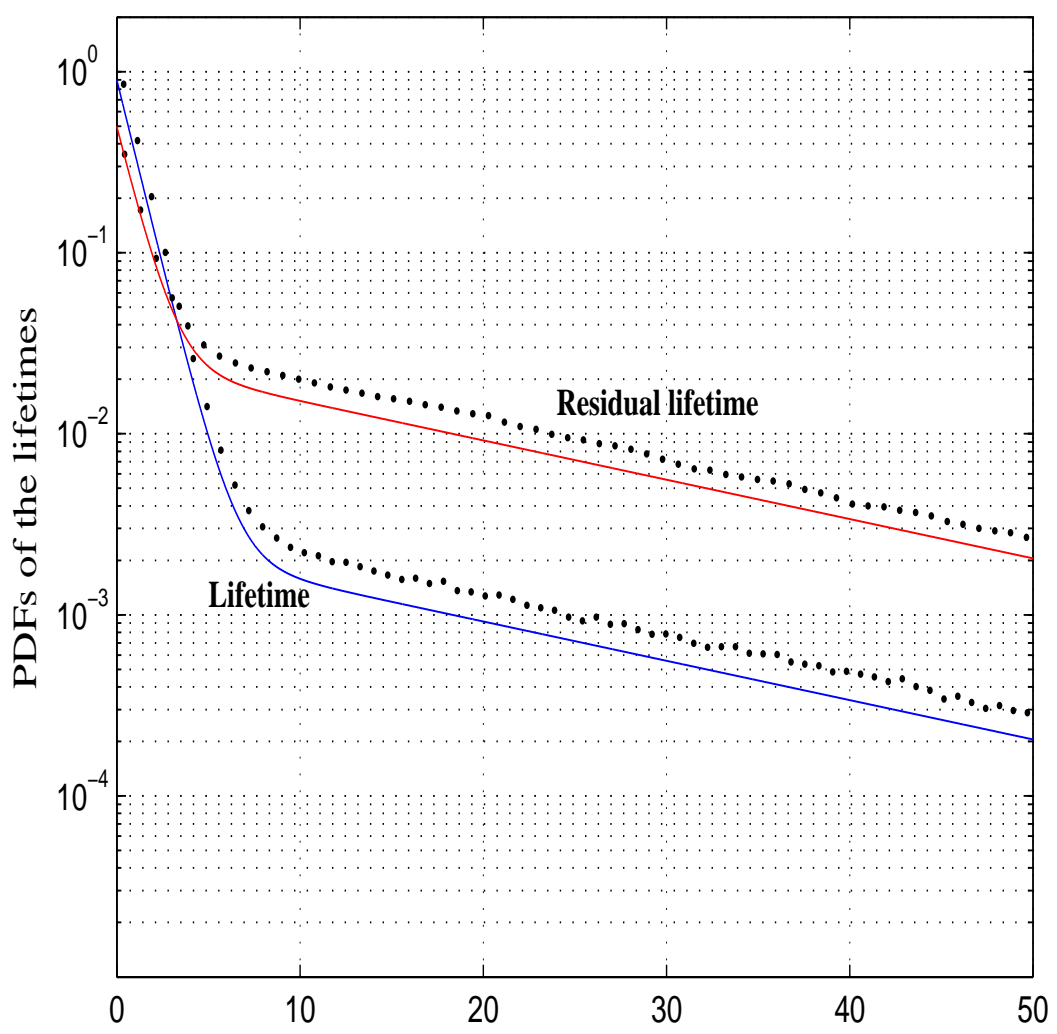

Figure 3: Probability density functions of the lifetime and of the residual lifetime in the case of long coherence time with $\alpha=0.9$. Points: experiment, continuous curves: theory. 